\title{
La cesura dottrinale di fine anni Trenta. Itinerari della giuspubblicistica italiana tra fascismo e Repubblica
}

\author{
Massimiliano Gregorio*
}

\section{Tra fascismo e Repubblica}

Quando Costantino Mortati, nel novembre del 1944, decise di iscriversi alla Democrazia cristiana, una volta inoltrata la richiesta, ricevette una lettera da Mario Scelba che poneva sub iudice la sua domanda contestando al grande giurista calabrese alcune sue prese di posizione contenute negli scritti pubblicati negli anni del regime che parevano esprimere, rispetto ad esso, una sconveniente benevolenza. Prescindendo dalla replica di Mortati ${ }^{1}$ con la quale egli difese le proprie posizioni dottrinali adducendo di aver sempre mantenuto ferma la propria opinione circa la necessità di un rafforzamento dell'esecutivo nell'orditura costituzionale (argomentazione che evidentemente dovette sembrare convincente al partito che poi si degnò di concedergli la tessera), l'episodio testimonia in maniera paradigmatica quanto complessa e articolata risultò la transizione tra fascismo e Repubblica, anche per uno dei più rilevanti protagonisti della dottrina giuspubblicistica. Del resto, non si può neppure biasimare troppo la classe dirigente democristiana per la scrupolosità dimostrata, giacché in quel conci-

Università degli Studi di Firenze.

1 Sulla risposta di Mortati e più in generale sull'intera vicenda, si veda F. Lanchester, La dottrina costituzionalistica italiana dal fascismo alla Repubblica, «Rivista AIC», 2, 2018, in particolare pp. 10 e sgg.

Massimiliano Gregorio, University of Florence, Italy, massimiliano.gregorio@unifi.it, 0000-0001-9129-7927 FUP Best Practice in Scholarly Publishing (DOI 10.36253/fup_best_practice)

Massimiliano Gregorio, La cesura dottrinale di fine anni Trenta. Itinerari della giuspubblicistica italiana tra fascismo e Repubblica, pp. 23-38, @ 2020 Author(s), CC BY 4.0 International, DOI 10.36253/978-88-5518202-7.04, in Piero Barucci, Piero Bini, Lucilla Conigliello (edited by), I mille volti del regime. Opposizione $e$ consenso nella cultura giuridica, economica e politica italiana tra le due guerre, (c) 2020 Author(s), content CC BY 4.0 International, metadata CC0 1.0 Universal, published by Firenze University Press (www.fupress. com), ISSN 2704-5919 (online), ISBN 978-88-5518-202-7 (PDF), DOI 10.36253/978-88-5518-202-7 
tato e drammatico frangente della storia italiana, il requisito minimo richiesto alla costruenda nuova classe politica del Paese, non poteva che essere quello di una cristallina patente antifascista.

Peraltro, il dubbio espresso da Scelba in quella lettera torna saltuariamente ad emergere ancora oggi, quando si affronta il tema spinoso dei percorsi intellettuali dei giuristi nel trapasso tra regime fascista e Italia repubblicana. Proviamo a formularlo, allora, questo dubbio. La questione che questo breve saggio intende affrontare può essere infatti così sintetizzata: come è possibile che i più robusti e convinti costruttori (teorici e pratici) della Costituzione democratica repubblicana - in primis Mortati e Crisafulli, ma potremmo aggiungervi anche altri rilevanti protagonisti quali Esposito, Lavagna ecc. - fossero gli stessi giuristi che solo pochi anni prima scrivevano sotto il regime fascista analizzandone l'architettura costituzionale e mostrando sovente, rispetto alle innovazioni in essa introdotte, un sincero interesse quando non addirittura un più o meno esplicito apprezzamento? A volte l'interrogativo affiora in termini assai più sbrigativi e brutali: ma questi nostri giuristi, insomma, erano fascisti oppure no? In tutta onestà conviene esplicitare che il lettore non troverà, al termine della presente riflessione, una risposta a tale domanda. E non tanto perché fornire una risposta sia difficile, quanto piuttosto perché è la domanda a risultare mal posta. Nelle pagine che seguono proverò dunque a dimostrare il perché.

\section{Un Metodhenstreit tutto italiano}

Anzitutto, per capire come sia possibile tenere assieme la produzione giovanile degli anni Trenta dei più giovani costituzionalisti italiani con il loro impegno - diretto o indiretto - volto a contribuire alla costruzione della democrazia repubblicana, bisogna sgomberare il campo da una possibile e troppo semplice risposta. Occorre cioè sfuggire alla tentazione di banalizzare quella transizione, riducendola ad una scelta di mero opportunismo o trasformismo politico. Sia chiaro: l'esigenza di rifarsi una verginità politica democratica dopo anni di acquiescenza (quando non addirittura di più o meno tacita adesione) al regime fascista era assai diffusa nell'Italia di quegli anni. E il ceto dei giuristi non ne fu certo esente. Ma nella vicenda dei giovani costituzionalisti italiani cresciuti negli anni Trenta, la loro transizione dal fascismo alla Repubblica si comprende assai meglio riannodando il filo del dibattito dottrinale. E, nel farlo, conviene ovviamente cominciare dall'inizio.

Qual era dunque lo stato dell'arte del dibattito giuspubblicistico italiano negli anni Venti e Trenta? Come è noto, a partire dagli ultimi vent'anni del secolo XIX, anche il nostro Paese aveva fatto registrare una fioritura di studi giuspubblicistici improntati ad un forte rinnovamento metodologico. E il maggior merito di questa operazione va ascritto a colui che più di ogni altro si spese per rifondare la giuspubblicistica italiana e per creare una vera e propria scuola giuridica nazionale: V.E. Orlando. Non è certo questa la sede utile a ricostruire gli aspetti fondamentali di quella svolta copernicana, le sue ascendenze e i suoi tratti di originalità. Qui è sufficiente sottolineare il fatto che l'operazione messa in 
campo da Orlando rispondeva, oltre che a ovvie ragioni scientifiche, ad un preciso obiettivo di politica del diritto: l'elaborazione di una originale teoria dello Stato di diritto italiano (una nostrana Rechtsstaatslehre) doveva infatti servire a rafforzare dal punto di vista teorico il giovane Stato italiano che, difeso dalle solide mura del diritto e accudito dalle premurose attenzioni dei giuristi, vedeva $\mathrm{i}$ propri attributi principali - la personalità giuridica, la sovranità intesa come suo naturale attributo, il governo di gabinetto - cristallizzati in un'architettura teorica sottratta alla temperie della lotta politica e alle possibili minacciose conseguenze che essa poteva riverberare sulla sfera istituzionale. Il prezzo da pagare, naturalmente, fu quello di tagliare i ponti che dovevano collegare lo Stato con la società sottostante, con la conseguenza che il primo finiva per autolegittimarsi secondo la celebre lezione hegeliana ${ }^{2}$, mentre la seconda si limitava a recitare il ruolo di mero presupposto sostanziale e di destinatario delle azioni del primo. Quando Mussolini giunse al potere, la scuola giuridica nazionale poteva dunque dirsi saldamente consolidata dal lavoro di molti prestigiosi interpreti (tra i principali, oltre a Orlando, vanno certo ricordati Oreste Ranelletti e Santi Romano, principali costruttori di quella versione dello Stato di diritto che va sotto il nome di Stato amministrativo ${ }^{3}$. Quale fu allora l'atteggiamento di questi giuristi di fronte all'avvento del fascismo? Pur consci dei diversi e articolati percorsi personali e senza poter neppure soffermarsi sui dettagli della vicenda, si possono tuttavia assumere come paradigmatici alcuni atteggiamenti che dimostrano come inizialmente buona parte degli esponenti di quella dottrina, che si ispirava ad un modello statocentrico ottocentesco di impronta tedesca, guardarono con simpatia alla possibilità di riaffermare l'autorità dello Stato e la saldezza del governo in un Paese scosso da violenti fermenti sociali (il celebre biennio rosso) e alle prese con nuovi equilibri politici (conseguenti alla riforma elettorale in senso proporzionale del 1919) tutti da trovare. La luna di miele tra il regime e alcune tra le più autorevoli voci del liberalismo italiano (oltre a Orlando anche Salandra accetterà ad esempio di aderire alla 'Lista nazionale' con la quale Mussolini si presentò alle elezioni del 1924) non durò tuttavia molto; giusto il tempo di permettere al duce del fascismo di virare decisamente, dopo l'omicidio Matteotti, verso una deriva dittatoriale che svuotava l'ordinamento statutario.

Da quel momento in avanti, ossia a partire indicativamente dal $1925^{4}, \mathrm{i}$ rapporti tra la scuola giuridica nazionale e il regime fascista cambiarono dra-

2 Cfr. G.W.F. Hegel, Lineamenti di filosofia del diritto (1821), Laterza, Roma-Bari 2004.

Cfr. L. Mannori, B. Sordi, Storia del diritto amministrativo, Laterza, Roma-Bari 2001.

4 Del 3 gennaio 1925 è il discorso di Mussolini alla Camera che annunciava la deriva autoritaria, poi tradotta in sostanza costituzionale dalle due leggi fascistissime (la L. 2263 del 24 dicembre 1925 e la L. 100 del 31 gennaio 1926) che stravolgevano gli equilibri costituzionali rafforzando enormemente la posizione dell'esecutivo e soprattutto quella del Capo del Governo (che cessava di essere così un primus inter pares rispetto ai Ministri), a discapito del Parlamento, svuotato di gran parte delle sue funzioni legislative e privato della possibilità di sfiduciare il vertice dell'esecutivo. È significativamente in quello stesso anno che V.E. Orlando si dimise da Deputato per ritirarsi a vita privata. 
sticamente e, per ricostruirli, occorre immergersi in profondità nel dibattito giuspubblicistico. Alla progressiva fascistizzazione dello Stato che Mussolini rapidamente realizzò imponendo una sempre più disinvolta confusione di piani tra Partito nazionale fascista e istituzioni statali ${ }^{5}$, la scuola giuridica nazionale rispose attestandosi su una posizione di ferma difesa delle proprie categorie concettuali e, quindi, del complessivo patrimonio dogmatico della Staatslehre europea, elaborata per larga parte nel secolo precedente. Ciò avvenne in maniera assai generalizzata, anche se attraverso percorsi molto differenti. Ad esempio: se V.E. Orlando optò per uno sdegnoso ritiro dalla scena pubblica (accompagnato anche da un sostanziale silenzio scientifico), Santi Romano al contrario scelse di continuare a giocare fino in fondo il ruolo di difensore delle categorie rechtsstaatlich all'interno del regime fascista, ponendosi così nella posizione di dover accettare numerosi ed imbarazzanti compromessi, prima da Presidente del Consiglio di Stato e poi da Senatore. In linea di massima, tuttavia, dal punto di vista scientifico gli esiti furono molto simili: la scuola giuridica nazionale si pose in una posizione di arrocco a difesa delle proprie categorie, che spesso e volentieri sconfinò in un colto formalismo completamente distaccato dalla realtà politica e costituzionale del regime.

Questo genere di atteggiamento, come è ovvio, attirò sulla dottrina di scuola liberale gli strali di un'altra fazione di giuspubblicisti: di coloro cioè che avevano invece sposato appieno la causa di Mussolini e si ponevano perciò come alfieri di una nuova rivoluzione metodologica, che avrebbe dovuto connotare in senso pienamente fascista anche la scienza italiana del diritto pubblico. «Se tutto una rivoluzione rivoluziona», si chiedeva polemicamente Sergio Panunzio, «è mai possibile che non si rivoluzionino anche i metodi e i concetti giuridici $\gg ?^{6}$ Del resto il fascismo non aveva forse preso le mosse da una severa critica verso l'imbelle ed impolitico Stato liberale? Non può dunque stupire che i suoi giuristi più in vista (oltre a Panunzio vanno certo ricordati Costamagna e Rocco, ma anche Chimienti, Maraviglia, Fragapane, Volpicelli ecc.) avvertissero la conseguente necessità di demolire le costruzioni teoriche che quella forma politica avevano contribuito a creare e a sostenere. I toni di questo scontro, tuttavia, furono particolarmente aspri. Come sempre accade, infatti, anche in quel caso le questioni meramente metodologiche celavano problematiche di ben altra so-

\footnotetext{
A mero titolo di esempio, oltre ai provvedimenti legislativi citati nella nota precedente, vale la pena ricordare: nel novembre 1926 la modifica del TULPS che consentì lo scioglimento dei partiti antifascisti e inaugurò quindi il regime a partito unico e, a venti giorni di distanza, l'introduzione del Tribunale speciale per la difesa dello Stato, competente a perseguire - con pene durissime compresa quella capitale - un buon numero di reati politici; nel 1927 il Gran consiglio del fascismo, massimo organo del PNF approvò la Carta del lavoro, poi immediatamente pubblicata - con assoluto dispregio di ogni attribuzione di competenze istituzionali - sulla Gazzetta ufficiale del Regno; nel 1928, infine il Gran consiglio venne addirittura trasformato in organo costituzionale dello Stato.

6 S. Panunzio, Lo Statuto del Partito, «Gazzetta del Mezzogiorno», 3 novembre 1929, ora in Id., Rivoluzione e Costituzione. Problemi costituzionali della rivoluzione, Treves, Milano 1933, p. 172.
} 
stanza. Il presupposto non esplicitato - ma perfettamente intellegibile (e ben lo compresero infatti anzitutto i giuristi engagé) - della difesa ad oltranza delle proprie categorie concettuali operata dalla scuola giuridica nazionale era infatti l'idea che lo Stato di diritto, lungi dall'essere una forma politica storicamente determinata, fosse invece il punto di arrivo ultimo e non più perfettibile (se non tramite puntuali e limitati aggiustamenti) del pensiero costituzionalistico moderno. In altre parole, gli epigoni di Orlando difendevano l'imprescindibilità delle categorie rechtsstaatlich perché ritenevano che imprescindibile, oltre che inevitabile, sarebbe stato prima o poi il ritorno al Rechtsstaat. Si trattava solo - per usare un'espressione cara alla commedia di De Filippo - di far passare la nottata, difendendo il difendibile, anche a prezzo di fornire un' interpretazione dell'esperienza costituzionale fascista completamente (e consapevolmente) scollegata dalla realtà.

Vale la pena fare qualche esempio per apprezzare la profondità della frattura scientifica e per comprendere l'atteggiamento della dottrina tradizionale di fronte alle innovazioni costituzionali apportate dal regime. Uno dei terreni di scontro più frequenti fu certamente incarnato dal partito politico. Il motivo è assai semplice: il partito, che per la Rechtsstaatslehre del secolo precedente era un soggetto sprovvisto di qualsiasi cittadinanza costituzionale, dal primo dopoguerra in poi aveva progressivamente assunto (e ciò era particolarmente evidente in Italia, visto che dal 1926 il PNF era divenuto partito unico) una posizione schiettamente pubblicistica 7 . Ciò nonostante, Santi Romano - ancora nel 1933, e quindi ben cinque anni dopo la trasformazione del Gran consiglio del fascismo da supremo organo collegiale del PNF a organo costituzionale di primaria importanza - insisteva nel negare che al Partito nazionale fascista fosse mai stata riconosciuta personalità giuridica ${ }^{8}$. L'argomentazione del grande giurista siciliano era di un formalismo disarmante: poiché la legislazione fascista aveva riconosciuto la personalità giuridica delle articolazioni territoriali del PNF e persino della Direzione del partito, ma nulla aveva detto circa il partito nel suo insieme, dal silenzio del legislatore non si poteva che dedurre una sua volontà negativa. Non che la questione avesse una qualche pratica rilevanza, sia chiaro: il ruolo pubblicistico del partito fascista e dei suoi vertici era sotto gli occhi di tutti. Ma fornisce comunque la misura della distanza tra le posizioni dottrinali e dell'uso - evidentemente politico, per quanto di politica del diritto si trattasse - che i giuristi di formazione liberale fecero del metodo e della dogmatica.

Come andò a finire dunque questo aspro confronto dottrinale, questo $\mathrm{Me}$ thodenstreit? Col senno di poi, possiamo certamente concludere che nessuna delle due parti in conflitto ne uscì vincitrice. La dottrina più vicina al regime non riuscì infatti a raggiungere l'obiettivo, troppo pretenzioso, di riscrivere in-

7 Sull'evoluzione delle interpretazioni giuspubblicistiche sulla natura e il ruolo costituzionale del partito politico, sia consentito rimandare a M. Gregorio, Parte totale. Le dottrine costituzionali del partito politico in Italia tra Otto e Novecento, Giuffrè, Milano 2013.

8 S. Romano, Corso di diritto costituzionale, Cedam, Padova 1933, in particolare p. 126. 
tegralmente le categorie giuspubblicistiche e rimettere pertanto in discussione l'edificio teorico eretto magistralmente da Orlando e dai suoi allievi. Così i giuristi di regime, pur riuscendo agevolmente a dimostrare l'inadeguatezza delle categorie rechtsstaatlich alla comprensione della realtà costituzionale fascista, non furono altrettanto capaci di sostituirle con convincenti alternative, finendo spesso - forse quasi sempre - col rifugiarsi invece nel metagiuridico, ossia nella potenza evocativa della retorica mussoliniana, tanto roboante quanto poco adeguata alla definizione concettuale. Di converso, tuttavia, neppure la scuola giuridica nazionale riuscì nell'intento che si prefiggeva. Certo, gli epigoni di Orlando riuscirono a difendere il proprio patrimonio dogmatico, che sopravvisse al regime fascista; ma alla caduta di quest'ultimo, non si verificò quell'ovvio ritorno allo Stato di diritto liberale, da molti di loro preconizzato e certo auspicato.

\section{La cesura di fine anni Trenta: una terza via dottrinale}

Come si è già avuto modo di spiegare in altri scritti' ${ }^{9}$, gli esiti di quel profondo conflitto dottrinale vennero dunque raccolti e messi a frutto da una nuova generazione di giovani costituzionalisti che, rispetto alla frontale contrapposizione tra scuola giuridica nazionale e giuristi engagé, ebbe il merito di aprire una terza via. I nomi di questi protagonisti sono noti: Mortati, Crisafulli, Esposito, Lavagna, Pierandrei, Zangara, M.S. Giannini e altri se ne potrebbero certo aggiungere. Come appare evidente a chiunque abbia qualche familiarità con le loro tesi, si tratta di un gruppo di autori assai eterogeneo e plurale; essi muovevano da presupposti teorici di partenza assai differenti e giunsero ad approdi interpretativi e financo politici non meno distanti, nella nuova stagione democratica e repubblicana ${ }^{10}$. Quindi non possono certamente essere considerati una scuola. Erano piuttosto accomunati da un dato generazionale e dalla condivisione di una nuova prospettiva di studi.

9 Cfr. Gregorio, Parte totale, cit. e, più recentemente, Id., Parte totale. Vincenzo Zangara e le dottrine del partito politico negli anni Trenta, «Nomos. Le attualità nel diritto», 3, 2018.

10 Basti pensare al fatto che, una volta caduto il regime, ognuno di questi giuristi divenne un riferimento intellettuale per aree politiche e culturali molto diverse: Mortati fu protagonista attivissimo nella DC, che lo candidò facendolo eleggere all'Assemblea costituente; Crisafulli divenne invece il costituzionalista di riferimento del PCI (sulla scelta del Partito comunista di non candidare Crisafulli all'Assemblea costituente, v. infra nota n. 41); M.S. Giannini fu capo di gabinetto socialista del Ministero per la Costituente guidato da Pietro Nenni; Esposito divenne un'ascoltata e rispettata voce dell'area liberale; mentre Zangara, dopo aver ricoperto anche il ruolo di vicesegretario del PNF, amava ironizzare nel dopoguerra sul fatto di essere stato epurato due volte: prima da Mussolini e poi dagli antifascisti. L'attenzione della dottrina sulle vicende di questi costituzionalisti continua a rimanere assai vivace. Tra i contributi più recenti si segnalano: il volume V. Crisafulli, Politica e Costituzione. Scritti "militanti" (1944-1955), a cura di S. Bartole e R. Bin, Franco Angeli, Milano 2018; mentre, per quanto concerne la poco studiata figura di Vincenzo Zangara, si veda il bel numero monografico a lui dedicato dalla rivista «Nomos. Le attualità nel diritto», 3, 2018. 
Dimostrare il primo aspetto è invero semplice: con l'unica eccezione di Mortati, che del gruppo era il più anziano essendo nato nel 1891, tutti gli altri erano figli del nuovo secolo. E, in quanto tali, avevano mosso i primi passi accademici e scientifici nel corso degli anni Trenta, spesso dopo essersi formati al magistero di giuristi vicini al regime (lo stesso Mortati aveva, dopo una prima laurea in giurisprudenza, preso una seconda laurea in scienze politiche come allievo di Sergio Panunzio). Quanto alla nuova prospettiva di studio, occorre riconoscere loro il merito di aver contribuito - collettivamente - ad aprire una terza via, inaugurando così un vero e proprio cambio di paradigma nello studio del diritto costituzionale. Ciò derivò dalla loro capacità di pervenire ad una sintesi innovativa rispetto a quell'aspra contesa metodologica sopra descritta, che li portò a non aderire in toto né alla difesa del patrimonio rechtsstaatlich né alle pretese di una revisione palingenetica della grammatica costituzionale auspicata dai giuristi di regime. Approdarono viceversa ad una soluzione che comportava il recupero e, al tempo stesso, il rifiuto di alcuni tratti caratterizzanti entrambi gli schieramenti in lotta, inaugurando così una nuova stagione di studi.

Che cosa recuperarono dalla scuola giuridica nazionale? Recuperarono la ferma convinzione dell'importanza e della bontà del metodo giuridico e, con essa, rifiutarono dunque quel continuo rifugiarsi nella sfera del metagiuridico, che era divenuto una cifra caratterizzante le riflessioni dei giuristi più vicini al regime. Nelle argomentazioni teoriche dei costituzionalisti cresciuti negli anni Trenta, infatti, non si cede mai alla facile tentazione di giustificare nuovi assetti costituzionali o nuove declinazioni di istituti giuridici con la comoda argomentazione della palingenetica rivoluzione fascista. Il ragionamento, al contrario, si snoda sempre entro i binari rigorosi della logica giuridica, ma senza che questo si traduca mai in una difesa aprioristica delle categorie ottocentesche che, anzi, uscivano da quelle analisi profondamente trasformate e svecchiate, e dunque più adeguate alla comprensione dei nuovi tempi. A ben vedere, peraltro, per apprezzare questo tipo di approccio spesso non è neppure necessario addentrarsi nella lettura dei testi, ma è sufficiente fermarsi alla scelta dei titoli. La prima celebre opera di Vezio Crisafulli, datata 1939, era infatti così intitolata: Per una teoria giuridica dell' indirizzo politico ${ }^{11}$. Ad uno sguardo poco attento, la sottolineatura dell'aggettivo «giuridico» nel titolo del saggio potrebbe passare inosservata o, al più, essere considerata una ridondante precisazione, figlia di un forse troppo scrupoloso zelo definitorio dell'autore. Ma non è così. E lo testimonia il fatto che Crisafulli non fu il solo a premurarsi di attribuire una patente di giuridicità, sin dal titolo, alla sua analisi. Pochi anni dopo, Carlo Lavagna intitolava un suo studio sugli assetti e le relazioni interne all'esecutivo Contributo alla determinazione dei rapporti giuridici fra Capo del Governo e Ministri ${ }^{12}$, mentre per uno

11 V. Crisafulli, Per una teoria giuridica dell' indirizzo politico, «Studi urbinati. Serie A», 13, 1939.

12 C. Lavagna, Contributo alla determinazione dei rapporti giuridici fra Capo del Governo e Ministri, Edizioni Universitarie, Roma 1942. 
scritto dello stesso anno sul tema della rappresentanza sceglieva il seguente titolo: Per un' impostazione dommatica del problema della rappresentanza politica ${ }^{13}$. «Teoria giuridica», ricostruzione «dogmatica», analisi dei rapporti « ci»: siamo di fronte ad un utilizzo deliberato di questi vocaboli, che vengono quasi ostentati a mo' di manifesto. Un utilizzo, vale la pena sottolinearlo, non solo non riscontrabile negli scritti dei giuristi più vicini al regime, ma che non ricorreva neppure nelle opere degli epigoni della scuola giuridica nazionale, $i$ quali cercavano di tenere invece un profilo assai più basso, probabilmente per evitare di offrire il fianco alle polemiche, già aspre, dei colleghi engagé. Ma ad ulteriore testimonianza della diffusione di una rinnovata attenzione agli aspetti dogmatici e metodologici è possibile citare anche altre evidenze. Nello stesso senso, possiamo leggere infatti la polemica tra Esposito e Mortati sulla determinazione dei concetti di nazione e di rappresentanza ${ }^{14}$, oppure l'altra celeberrima querelle, sviluppatasi anch'essa sulle colonne della rivista «Stato e diritto» nel 1940, tra Vezio Crisafulli e Giuseppe Maranini sul metodo nello studio del diritto costituzionale ${ }^{15}$. Dunque, sottolineare già nei titoli delle opere la ferma volontà di indagare i temi oggetto dell'analisi con gli strumenti tecnici della dogmatica giuridica rispondeva ad una sorta di presa di posizione pubblica, che rimarcava una continuità con la riflessione giuspubblicistica antecedente al regime e, va da sé, una corrispondente presa di distanza dalle posizioni dei giuristi più vicini al fascismo. Questo secondo aspetto, anzi, finisce per essere quello che viene maggiormente in evidenza quando si vanno a considerare le conseguenze che la ricerca di una terza via rispetto al Methodenstreit proiettava sul piano dei contenuti. Vale la pena sottolineare, infatti, come il recupero del metodo giuridico dell'analisi finiva per tradursi, in molti casi, anche nel recupero di una dimensione propria del giuridico che, negli ambienti della dottrina più prossima al duce, non godeva più, per ovvie ragioni, di grande credito. Ci si riferisce cioè al diritto inteso come limite, alla funzione di garanzia intimamente connaturata alla dimensione del giuridico. Anche questo aspetto può essere considerato un elemento di continuità con la dottrina ottocentesca, visto che lo Stato di diritto nasceva proprio dall'idea che il superamento degli assetti politici di antico regime passasse, anzitutto, dalla giuridicizzazione dei rapporti tra autorità e individui. Formalizzare e proceduralizzare le forme dell'agire statuale metteva infatti in condizione i cittadini di poterne prevedere gli esiti e la prevedibilità dell'azione statuale risultava così essere uno dei principali tratti della

13 C. Lavagna, Per una impostazione dommatica del problema della rappresentanza politica, «Stato e diritto», 3 (3), 1942.

14 Esposito dedicò al tema almeno due scritti: C. Esposito, Lo Stato e la Nazione italiana, «Archivio di diritto pubblico», 1937 e Id., Lo Stato fascista, 1940. Alle critiche di Mortati, espresse soprattutto in C. Mortati, Sulla posizione del partito nello Stato, «Stato e diritto», 2 (4-5) 1941, Esposito rispose con il saggio Lo Stato nazionale fascista, «Stato e diritto», 3 (3), 1942.

15 Cfr. G. Maranini, Qualche osservazione di metodo sugli studi di diritto costituzionale, «Stato e diritto», 1 (1), 1940, e l'immediata replica di V. Crisafulli, Ancora a proposito del metodo negli studi di diritto costituzionale, «Stato e diritto», 1 (2), 1940, pp. 122-127. 
Rechtsstaatslehre ottocentesca. E dunque, nonostante il marcato antiformalismo dei giuristi engagé, la dimensione garantistica del diritto tornò invece ad emergere decisamente negli scritti dei giovani costituzionalisti cresciuti negli anni Trenta. Un esempio può essere utile. Quando Crisafulli e Lavagna si interrogarono sull'indirizzo politico, le loro analisi non miravano a sostenere tanto il ruolo, per molti aspetti nuovissimo, dell'istituto, che si sostanziava nella posizione di un principio guida in grado di permeare l'intera azione dello Stato. Al contrario, essi si concentrarono sulle articolazioni interne al concetto e sui limiti che esso doveva necessariamente incontrare, distinguendo così, per esempio, tra indirizzo politico di governo e indirizzo politico generale dello Stato ${ }^{16}$, oppure specificando - come fece ancora Crisafulli - che il grado di vincolatività giuridica dell'indirizzo politico subiva modificazioni importanti a seconda dei destinatari del vincolo medesimo. Per cui se l'indirizzo politico certo impegnava in massimo grado l'azione delle amministrazioni più periferiche dello Stato, diverso doveva essere il ragionamento da applicare agli organi costituzionali. Anche questi erano sottoposti al vincolo dell'indirizzo politico, ma l'intensità di quel vincolo non poteva mai spingersi fino al punto da compromettere l'autonomia di valutazione che la posizione apicale di tali organi nell'ordinamento costituzionale necessariamente comportava.

Si è detto, sin qui, di quanto la nuova generazione di costituzionalisti riprese dalla dottrina tradizionale, quella di scuola orlandiana. Ma cosa trasse invece dalla dottrina più vicina al regime? Certamente i temi. Su che cosa indagavano infatti i giovani giuspubblicisti cresciuti negli anni Trenta? Vale la pena soffermare l'attenzione su questo punto. Scorrendo brevemente le opere comparse tra la fine di quel decennio e i primi anni del nuovo, è possibile individuare diversi temi ricorrenti: si occuparono di indirizzo politico Mortati nel $1931^{17}$ e Crisafulli nel $1939^{18}$; di partito politico Zangara nel $1935^{19}$, nel $1938^{20}$ e nel $1939^{21}$, Mortati nel 1941 ${ }^{22}$; di rappresentanza Zangara nel 1938 e $1939^{23}$, Esposito nel $1939^{24}$ (ma anche negli scritti tra il 1937 e il $1940^{25}$ ) e Lavagna nel

16 Così Crisafulli, che anticipava evidentemente la futura dialettica tra indirizzo politico di maggioranza e indirizzo politico costituzionale.

17 C. Mortati, L'ordinamento del governo (1931), Giuffrè, Milano 2000.

18 Crisafulli, Per una teoria giuridica dell' indirizzo politico, cit.

19 V. Zangara, Il partito e lo Stato, SEM, Catania 1935.

20 V. Zangara, Il partito unico e il nuovo Stato rappresentativo in Italia e in Germania, Zanichelli, Bologna 1938.

21 V. Zangara, Il Partito Nazionale Fascista, in Nuovo Digesto Italiano, vol. IX, Utet, Torino 1939.

22 Mortati, Sulla posizione del partito nello Stato, cit.

23 Zangara, Il partito unico e il nuovo Stato rappresentativo, cit., nonché Id., La rappresentanza istituzionale, Zanichelli, Bologna 1939.

24 C. Esposito, La rappresentanza istituzionale, Filelfo, Tolentino 1939.

25 Si veda sopra la nota $\mathrm{n} .11$. 
$1942^{26}$. Vi fu infine chi tentò una ricostruzione decisamente più ampia e ambiziosa attorno al concetto di costituzione, come fece Mortati nel $1940^{27}$, peraltro mettendo a frutto altre prodromiche intuizioni quali quelle sul concetto di regime ${ }^{28}$ prodotte da Chiarelli già nel $1932^{29}$, ancora da Zangara nel $1935^{30}$, da Gueli nel $1939^{31}$. Ora, alla luce dell'evidente consonanza delle riflessioni su temi di ricerca chiaramente ricorrenti, è opportuno chiedersi che cosa avessero in comune questi temi tra di loro. E la risposta è straordinariamente semplice: tutti questi concetti (o per meglio dire istituti) si collocavano su una direttrice ben precisa. Erano cioè tutte tappe di un unico percorso: quello che - per usare le parole di Zangara nel 1938 - mirava a consentire al «popolo di partecipare alle funzioni sovrane dello Stato ${ }^{32}$. Ora, è proprio questo aspetto a segnare, come è evidente, una profonda discontinuità con la scuola giuridica nazionale. La Rechtsstaatslehre ottocentesca aveva proceduto, come accennato in precedenza, ad una ricostruzione del rapporto tra Stato e società improntato ad una nettissima separazione, mirata anzitutto ad escludere, in capo al corpo sociale, ogni tipo di pretesa legittimante l'ordine costituzionale. Emblematica, da questo punto di vista, risultava la teorizzazione della rappresentanza politica nella ricostruzione di V.E. Orlando, per il quale l'atto del votare non era in alcun modo configurabile come esercizio di un diritto. L'interesse che quell'atto mirava a soddisfare, infatti, non era mai l'interesse del singolo elettore (magari a veder rappresentata la propria opinione), ma - al contrario - l'interesse dello Stato a che un suo organo particolarmente rilevante fosse composto da persone capaci e adeguate al ruolo. Era dunque lo Stato ad investire di una funzione pubblica, quella della scelta dei soggetti più idonei all'ufficio, l'elettore; e la posizione di quest'ultimo era pertanto quella di un soggetto chiamato ad adempiervi. Non a caso, notava Orlando, lo Stato non chiedeva ausilio a tutti i cittadini, ma li sele-

26 Lavagna, Per una impostazione dommatica del problema della rappresentanza politica, cit.

27 L'ovvio riferimento è a C. Mortati, La costituzione in senso materiale, Giuffrè, Milano 1998 (1940). Per un approfondimento sul tema si rinvia a M. Fioravanti, Dottrina dello Statopersona e dottrina della costituzione. Costantino Mortati e la tradizione giuspubblicistica italiana, in Id., La scienza del diritto pubblico. Dottrine dello Stato e della costituzione tra Otto e Novecento, Giuffrè, Milano 2001, t. II, pp. 657 e sgg., nonché ancora M. Fioravanti, Il concetto di costituzione in senso materiale, «Historia constitucional», 12, 2011.

28 Sul punto sia consentito rimandare a M. Gregorio, L'idea di costituzione nella giuspubblicistica italiana degli anni Trenta, in La costruzione della 'legalità' fascista negli anni Trenta, a cura di I. Birocchi, G. Chiodi, M. Grondona, Roma Tre press, Roma 2020, in particolare pp. 189 e sgg.

29 G. Chiarelli, Il concetto di "Regime" nel diritto pubblico, «Archivio giuridico Filippo Serafini», 1932.

30 Zangara, Il partito e lo Stato, cit.

31 V. Gueli, Il regime politico nello Stato fascista, R. De Luca, Roma 1939.

32 Zangara, Il partito unico e il nuovo Stato rappresentativo, cit., p. 40. 
zionava rivolgendosi soltanto a coloro che, soddisfacendo certi requisiti, si mostravano degni di possedere una capacità elettorale ${ }^{33}$.

Se quindi il secolo XIX aveva potuto permettersi di non prendere neppure in considerazione il problema della partecipazione popolare alla determinazione delle scelte dello Stato, questo tema emerge invece nel Novecento in tutta la sua dirompente urgenza. Potremmo anzi dire di più. La questione del come consentire al popolo di innervare lo Stato di contenuti positivi non era una delle tante questioni poste dal Novecento giuridico, ma al contrario la questione più delicata e centrale che la cultura giuridica del secolo XX si era trovata ad affrontare. E, sia detto per inciso, il movimento fascista - che fu certo tante cose, ma tra queste fu anche, e senza alcun dubbio, un figlio legittimo del Novecento - non si sottrasse all'onere di fornire una propria risposta al problema. Certo, fu una non soluzione, ossia una risposta fittizia e di comodo, più propensa ad eludere il problema che a risolverlo, una risposta antidemocratica e drammaticamente semplicistica, ma fu una risposta. Senza alcuna pretesa di esaustività e, anzi, unicamente per offrirne un' immagine schematizzata, possiamo dire che la soluzione offerta dal regime fascista al problema della partecipazione politica del popolo alla determinazione delle scelte politiche statuali si sostanziò in un tautologico ragionamento di chiaro stampo organicistico. Poiché per il fascismo degli esordi risultò politicamente assai fruttuoso presentarsi al Paese come unico movimento votato a perseguire non fini di parte, ma il superiore interesse della nazione, sin dalla sua costituzione in partito - avvenuta nel 1921 - decisero di trarne le conseguenze più radicali: il fascismo cioè si identificò - $o$ almeno pretese di farlo - con la nazione. Lo rivela chiaramente anche l'ordine col quale si scelsero gli aggettivi chiamati a caratterizzare il nuovo partito, che era prima nazionale e solo dopo fascista. E dunque, una volta giunto al potere, fu piuttosto naturale per il regime, che si presentava come l'incarnazione istituzionale della riscossa rivoluzionaria promossa dalla migliore gioventù italica, attrezzare dei canali permanenti (il Partito nazionale fascista appunto e l'ordinamento corporativo) che avrebbero dovuto consentire al pluralismo sociale - tollerato fintanto che non avesse imboccato derive antinazionali, ossia antifasciste - di alimentare costantemente quel circuito virtuoso volto a determinare le scelte strategiche dell'agire dello Stato. In teoria, dunque, il fascismo aspirava a costruire una sorta di articolato meccanismo di integrazione del popolo nello Stato, simile a quello teorizzato da Smend nella sua Integrationslehre ${ }^{34}$. In pratica, tuttavia, la centralità assunta dall' italianissimo Führerprinzip (sui rischi del quale peraltro Smend aveva espressamente richiamato l'attenzione) che riconduceva l'intera catena di comando al duce del fascismo aveva finito per trasformare questo cir-

33 Cfr. V.E. Orlando, Del fondamento giuridico della rappresentanza politica (1895), ora in Id., Diritto pubblico generale. Scritti varii (1881-1940) coordinati in sistema, Giuffrè, Milano 1954; nonché V.E. Orlando, Principii di diritto costituzionale, Barbera, Firenze 1889.

34 Cfr. R. Smend, Verfassung und Verfassungsrecht, Duncker und Humblot, München-Leipzig 1928. 
colare processo di integrazione (di principio corporativo parleranno alcuni entusiasti interpretati come Volpicelli) in un meccanismo del tutto verticale in cui le direttive partivano dal centro e giungevano alla periferia del sistema ${ }^{35}$. Del resto, tra le tante ambiguità che caratterizzarono la costruzione teorica fascista vi era anche quella - e non era certamente tra le meno problematiche - che pretendeva di tenere assieme la narrazione di una rivoluzione popolare nata dal basso con una struttura di potere spiccatamente statualistica, ben cristallizzata nel celebre slogan mussoliniano del «tutto nello Stato, niente al di fuori dello Stato, nulla contro lo Stato» ${ }^{36}$.

\section{La cesura di fine anni Trenta: un ponte tra fascismo e democrazia}

A ben vedere, fu proprio nello spiraglio teorico lasciato aperto dalla predetta ambiguità che si inserirono le innovative teorie costituzionali della nuova generazione di costituzionalisti. Quando questi tornarono a ricostruire il rapporto tra società e Stato sulla base della primazia della prima sul secondo non vennero tacciati di eresia perché, in fondo, anche tra i giuristi più vicini al regime esisteva già una vivace dialettica tra interpretazioni rigidamente stato-centriche (come quella di Costamagna) e interpretazioni invece più orientate in senso sociocentrico (quale quella di Panunzio, che proveniva dal mondo del sindacalismo rivoluzionario). I nostri giuristi, dunque, nel momento in cui teorizzavano il ruolo proattivo della società nel processo di determinazione dei fini politici dello Stato non descrivevano effettivamente la realtà dello Stato fascista, ma certo sostenevano un principio che un regime che pretendeva di incarnare il superiore interesse della nazione non avrebbe potuto non sottoscrivere. Naturalmente, non sfuggirà che il medesimo principio rappresentava - e ha poi effettivamente rappresentato - la pietra angolare di ogni forma politica democratica.

Il che ci riporta alla questione principale, dalla quale avevamo preso le mosse in avvio, ossia alla domanda sul come riuscì la generazione di costituzionalisti cresciuti negli anni Trenta ad elaborare, sul finire del decennio, interpretazioni costituzionali che potevano adattarsi alla dittatura fascista ma poi, caduta finalmente quest'ultima, risultarono perfettamente in grado di spiegare la realtà della

35 Naturalmente sbaglieremmo a pensare che la catena di comando del regime costruita per ricondurre tutto a Mussolini, comportasse la totale esclusione di ogni altro attore nei processi decisionali. Sulla perdurante rilevanza di specifici interessi di categoria, sulle politiche di potere personali portate avanti da alcuni gerarchi, sul persistere di una burocrazia ancora legata alla cultura del secolo precedente e su quanto il regime, in generale, fu costretto a scendere a patti senza riuscire nell'intento di rifondare integralmente la macchina statuale si è recente soffermato $\mathrm{G}$. Melis, La macchina imperfetta. Immagine e realtà dello Stato fascista, il Mulino, Bologna 2018. Ciò nonostante, pur consci della relatività insita in ogni interpretazione che abbia pretese modellizzanti, ci pare che l'assoluta centralità della figura monocratica del capo del governo e duce del fascismo giustifichi la ricostruzione sopra esposta, almeno sotto il profilo della teoria dello Stato.

36 La frase venne pronunciata da Mussolini in un discorso tenuto a Milano il 28 ottobre 1925, in occasione del terzo anniversario della marcia su Roma. 
nuova Italia democratica e repubblicana. La risposta sta nella peculiare prospettiva di analisi che essi assunsero: assai poco interessati a proporre interpretazioni esegetiche dell'ordinamento costituzionale fascista, essi si incamminarono sul più impegnativo ma anche più accattivante sentiero della teoria generale dello Stato e della costituzione (a dire il vero più della seconda che del primo). Non si trattò però di una fuga, cioè di un escamotage utile a rendere le proprie analisi più teoriche e rarefatte per sfuggire ad un eventuale censura del regime. Al contrario, negli scritti dei vari Mortati, Esposito, Crisafulli i riferimenti alla realtà dell'ordinamento fascista sono ben presenti. $\mathrm{E}$ anzi: questi costanti riferimenti vengono utilizzati proprio per corroborare la bontà delle tesi sostenute. Il che risultava possibile perché, nel tentativo di elaborare una teoria del nuovo Stato contemporaneo (che per ognuno di loro era lo Stato del secolo XX, in antitesi allo Stato di diritto liberale ottocentesco), i nostri autori considerarono lo Stato fascista come una declinazione di quella nuova tipologia di Stato. Una declinazione, per l'appunto; non l'unica possibile.

A conti fatti, è proprio questa ricollocazione dell'esperienza costituzionale fascista tra le tappe del lungo e glorioso percorso evolutivo del concetto di Stato moderno (che si fa classicamente cominciare con la rivoluzione francese, benché sempre più numerose interpretazioni tendano ad anticiparne il momento genetico al Cinque-Seicento ${ }^{37}$ ) a rappresentare la chiave di lettura più adeguata per rispondere alla nostra iniziale domanda e a spiegare, dunque, la cesura di fine anni Trenta. Naturalmente, questo costituisce però anche l'aspetto più problematico e controverso delle analisi dei nostri giuristi, come emerse con forza nel secondo dopoguerra, più che sul finire degli anni Trenta. Il che è, almeno in parte, perfettamente logico. Al regime non dovette dispiacere infatti di essere ascritto a buon diritto tra le tappe di una tanto nobile linea evolutiva e ciò probabilmente fece passare in secondo piano il corollario più dirompente di questa proposta di sistemazione, ossia la possibilità di pensare la modernità statuale in forme anche diverse da quelle dell'ordinamento costituzionale mussoliniano. Assai più problematico, invece, fu l'accettare questa imbarazzante parentela novecentesca tra dittatura e democrazia nel secondo dopoguerra. Lo stesso Mortati, come notava Gustavo Zagrebelsky nella prefazione alla ristampa de $L a$ costituzione in senso materiale per la Biblioteca per la storia del pensiero giuridico moderno ${ }^{38}$, infatti, evitò accuratamente (e comprensibilmente) di sottolineare, almeno nell'immediato secondo dopoguerra, i profili di continuità del suo pensiero. Tanto che non è dato rinvenire neppure l'espressione «costituzione in senso materiale» nei primissimi scritti pubblicati in età repubblicana, quali ad esempio quello sulla Costituente o nella prima edizione del suo fortunatis-

37 Al proposito, si vedano le pionieristiche intuizioni di Fioravanti sul cosiddetto « Stato giurisdizionale» contenute nel saggio Stato e Costituzione, in Lo Stato moderno in Europa. Istituzioni e diritto, Laterza, Roma-Bari 2002, a cura dello stesso M. Fioravanti.

38 C. Mortati, La costituzione in senso materiale, Giuffrè, Milano 1998 con una introduzione di G. Zagrebelsky. 
simo manuale di istituzioni di diritto pubblico ${ }^{39}$. Non vi fu nessuna abiura, sia chiaro. Semplicemente, i tempi sconsigliavano di mostrare continuità (fossero anche solo intellettuali o teoretiche), richiedendo invece, a gran voce, aperte e profonde discontinuità politiche. Il problema era dunque generale ${ }^{40}$, ma che per i giuristi si ponesse in modo più marcato e con tratti decisamente peculiari ${ }^{41} \mathrm{fu}$ immediatamente chiaro sin dai lavori dell'Assemblea costituente. Eccezion fatta per quei pochi giuristi che avevano avuto un ruolo attivo nell'antifascismo e nell'esperienza resistenziale (come ad esempio Piero Calamandrei) o che appartenevano al «mondo di ieri» (espressione che Paolo Grossi utilizza sovente, mutuandola da un celebre libro di Peter Zweig, per indicare la cultura giuridica ottocentesca ${ }^{42}$ di cui il principale esponente fu senza dubbio Vittorio Emanuele Orlando), nei confronti di tutti gli altri l'assemblea mostrò spesso una spiccata diffidenza. L'esempio paradigmatico e più efficace di tale atteggiamento è senz'altro rappresentato dal celebre j'accuse togliattiano rivolto proprio contro i giuristi e contenuto nel suo intervento dell'11 marzo 1947, nella seduta pomeridiana dell'Assemblea plenaria, chiamata a discutere il progetto di Costituzione. Vale la pena leggerlo per intero.

Oserei dire che nel nostro lavoro non ci hanno dato grande aiuto i giuristi. Non se l'abbiano a male i colleghi che esercitano questa nobile professione, che del resto avrebbe potuto anche essere la mia, se la politica non mi avesse traviato. Molte formulazioni del progetto sono certamente deboli, perché giuridicamente non siamo stati bene orientati e effettivamente fu un errore non includere nella Commissione i rappresentanti della vecchia scuola costituzionalista italiana. La realtà è però che negli ultimi venti o trenta anni la scienza giuridica si è staccata dai principî della nostra vecchia scuola costituzionale. [...] e questo spiega perché, quando abbiamo dovuta scrivere una Costituzione democratica e abbiamo chiesto l'ausilio dei giuristi, essi non sono stati in grado di darci un aiuto efficace. Per darcelo, occorreva ch'essi cancellassero o dimenticassero qualche

C. Mortati, La Costituente. La teoria, la storia, il problema italiano, Darsena, Roma 1945 e Id., Corso di istituzioni di diritto pubblico. Anno accademico 1948/49. Appunti dalle lezioni, Cedam, Padova 1949. Sul punto cfr. G. Zagrebelsky, Premessa in C. Mortati, La costituzione in senso materiale, cit., p. XX.

40 Paradigmatica al riguardo fu la vicenda del processo di epurazione, assai complicato da realizzare e capace di ottenere risultati assai limitati, sul quale tuttavia permangono opinioni e interpretazioni assai divergenti. Cfr., ad esempio, R. Palmer, Processo ai fascisti 1943-1948. Storia di un'epurazione che non c'è stata, Rizzoli, Milano 1996 e, sul versante opposto, H. Voller, I conti col fascismo. L'epurazione in Italia 1943-1948, il Mulino, Bologna 1997.

${ }^{41}$ La transizione tra fascismo e Repubblica fu per i giuristi particolarmente problematica (e per i costituzionalisti lo fu ancora di più) perché non si limitava a chiamare in causa scelte personali, ma incideva assai più in profondità sull'onestà intellettuale e sulla coerenza interpretativa di tutti coloro che continuarono a lavorare, a scrivere e ad insegnare sotto il regime. Cfr. ad esempio il celeberrimo saggio di A.C. Jemolo, Confessioni di un giurista, Giuffrè, Milano 1947.

42 P.Zweig, Il mondo di ieri. Ricordi di un Europeo (1941), Mondadori, Milano 1994. Sull'utilizzo che Grossi fa dell'espressione si veda ad esempio il recente P. Grossi, Il diritto in una società che cambia. A colloquio con Orlando Roselli, il Mulino, Bologna 2018, p. 111. 
cosa; bisognava che ritornassero a qualche cosa che avevano dimenticato, e non erano sempre in grado di farlo ${ }^{43}$.

L'analisi di Togliatti è spietata e draconiana. Il Segretario del PCI divideva il ceto dei giuristi in vecchia scuola e nuova generazione. Alla prima (e cioè a Orlando e i suoi epigoni), portatrice di sani principi giuridici, egli rimproverava al massimo il fatto di non aver saputo opporre al fascismo un argine che forse avrebbe evitato al Paese la sciagura della dittatura. Ma era la seconda a costituire il vero problema, perché i giuspubblicisti più giovani, cresciuti nel clima antidemocratico del ventennio, erano ritenuti strutturalmente incapaci di fornire ai padri e alle madri costituenti il contributo di sapere giuridico necessario alla stesura della nuova carta. In verità l'analisi togliattiana era clamorosamente e duplicemente sbagliata. In primis perché addebitava ai giovani costituzionalisti 'colpe' teoriche (quali la dottrina della sovranità dello Stato o la concezione dei cosiddetti Reflexrechte) in realtà imputabili proprio alla vecchia scuola liberale. $\mathrm{E}$, in secondo luogo, perché non si rendeva conto che era proprio quest'ultima, dati i differenti presupposti concettuali di partenza, a risultare assolutamente inadeguata a fornire conforto tecnico e teoretico al lavoro del costituente italiano. Ma ai fini del nostro ragionamento tutto ciò non pare particolarmente rilevante. Importa invece sottolineare come i percorsi teorici dei costituzionalisti cresciuti negli anni Trenta apparivano di per sé, verrebbe quasi da dire ontologicamente, sospetti ${ }^{44}$. E questo alone di sospetto ha faticato molto a dissiparsi; e forse non si è mai del tutto dissolto. I motivi sono vari, ovviamente, ma su uno almeno vale la pena soffermare l'attenzione, a chiosa di questa analisi.

Si tratta di una considerazione di carattere generalissimo, che chiama in causa anche la complessiva difficoltà italiana nel fare i conti col passato fascista. Senza la pretesa di addentrarsi in analisi che valicano decisamente le competenze di chi scrive, sia per lo meno consentito sottolineare che uno dei motivi di tale difficoltà sembra essere stata la diffusione per molti anni nel discorso pubblico, di un'immagine negativamente (e ciò è ovvio) parentetica (e ciò lo è assai meno) del ventennio fascista. Pur con tutte le migliori intenzioni, considerare il fascismo come un incidente della storia, come un buco nero tra un prima (retrò ma dotato di una sua nobiltà) e un dopo (identificato con la consacrazione della modernità democratica) conduce infatti inevitabilmente alla rimozione di quei vent'anni dalla storia della cultura giuspolitica italiana. Col risultato che tutto quanto è stato vissuto (e riflettuto e teorizzato) in quel periodo sembra legittimamente liquidabile sulla base di una aprioristica patente di indegnità. La cesu-

43 P. Togliatti, intervento nella seduta pomeridiana dell'11 marzo 1947, in Atti dell'Assemblea costituente ora reperibili in rete sul sito della Camera dei Deputati.

44 Emblematica al proposito la vicenda di Vezio Crisafulli che nel secondo dopoguerra si avvicinò al PCI fino a diventarne di fatto il giurista di riferimento per le questioni costituzionali. Ciò nonostante, come detto, proprio per il fatto di appartenere a quella generazione di giuristi cresciuti sotto il regime fascista, il PCI non lo candidò all'Assemblea costituente, della quale Crisafulli - ligio alla disciplina di partito - seguì i lavori dall'esterno riferendone ai lettori comunisti sulle colonne del periodico «Rinascita». 
ra di fine anni Trenta, invece, sta a dimostrare l'esatto contrario. E cioè che del Novecento giuridico si possono dare due differenti declinazioni: una democratica e una anti-democratica; e che tra le due versioni vi sono elementi di continuità tanto numerosi almeno quanto lo sono gli elementi di discontinuità. Non è disconoscendo le parentele novecentesche tra cultura costituzionale fascista e cultura costituzionale democratica che si tutela più saldamente la seconda. E poiché sembra assai convincente la tesi secondo la quale siamo ancora oggi ben dentro il lunghissimo Novecento giuridico ${ }^{45}$, è proprio nella vigile consapevolezza dell'esistenza di un'eventualità antidemocratica che si può progredire sulla strada della costruzione di una sempre più efficiente democrazia costituzionale.

In questo percorso, naturalmente, anche la scienza del diritto costituzionale deve fare la sua parte. Se i giovani giuspubblicisti cresciuti negli anni Trenta poterono, sul finire del decennio, preparare il terreno teorico per la transizione dal fascismo alla democrazia, ciò avvenne perché essi accettarono di indagare i presupposti più profondi della materia e si spinsero dunque fino alle radici della scienza medesima, al suo momento genetico, là dove società e istituzioni si toccano e si fondono, dove il politico diviene giuridico. Nell'introduzione alla già citata ristampa de La costituzione in senso materiale di Mortati, Gustavo Zagrebelsky citava un episodio assai illuminante. In un convegno di costituzionalisti italiani, nel 1994, un oratore che aveva espressamente dichiarato che, a suo avviso, la tesi mortatiana della costituzione in senso materiale sarebbe stata da abbandonare totalmente, ricevette per ciò solo un applauso a scena aperta. Segno evidente, notava Zagrebelsky, di «uno stato d'animo, un disagio $\gg^{46}$ evidentemente diffuso, che fece consumare, in quell'occasione «un vero e proprio evento dell'inconscio», una collettiva rimozione in senso freudiano ${ }^{47}$. La conclusione che il prefatore vent'anni fa trasse da quell'evento pare rappresentare ancora oggi un monito valido per il giurista contemporaneo: «quell'applauso è $[\ldots]$ un segno rivelatore che la dottrina della costituzione in senso materiale, con le questioni essenziali ch'essa ha sollevato e con le risposte che ha tentato di dare, è sempre ancora là a turbare la quiete [della] scienza costituzionalistica», e dunque a stimolarla a non perdere «interesse per l'investigazione delle proprie radici e dei propri fondamenti e quindi anche per le grandi domande che ne mettono in gioco il significato nel momento storico presente $\gg^{48}$.

45 Sul lungo Novecento giuridico si veda P. Grossi, Novecento giuridico. Un secolo pos-moderno. Discorso inaugurale per l'anno accademico 2010/2011 tenuto nell'Università di Ferrara, il 22 novembre 2010, ora in ID., Introduzione al Novecento giuridico, Laterza, Roma-Bari 2012.

46 Zagrebelsky, Premessa, cit., p. VII.

47 Ivi, pp. VIII-IX.

48 Ivi, p. IX. 